\title{
Effect of solubility enhancement methods, in-vitro release and stability studies of indomethacin
}

\begin{abstract}
The objective of present work was to compare the effect of different solubility enhancement methods like solvent dispersion, physical mixture, hydrotropy, solid dispersion and freeze drying on in-vitro release and stability studies of indomethacin. Indomethacin, BCS class II NSAIDs, acts by inhibiting COX-I and II enzymes and uses in treating various conditions like rheumatoid arthritis, pain and headache. Inclusion complex formulations were prepared using different methods like solvent dispersion, physical mixing, hydrotropy, solid dispersion and freeze drying. Particle size and zeta potential observed varied range of values between $479.4 \pm 1.12$ to $1417 \pm 2.37 \mathrm{~nm}$ and in between $-15.3 \pm 1.08$ to $-24.9 \pm 1.18 \mathrm{mV}$ respectively. The highest solubility was observed in solid dispersion due to highest reduction of particle size. In-vitro drug release study showed controlled indomethacin release pattern in solid dispersion for $24 \mathrm{~h}$ as compared to other inclusion complexes. Solid dispersion method emphasizes its importance in enhancing the solubility and bioavailability, controlled release and stability of indomethacin.
\end{abstract}

Keywords: solubility enhancement, indomethacin, $\beta$-cyclodextrin, solid dispersion
Volume 3 Issue 7 - 2016

\author{
Pravin K Shende, Gaud RS, Foram Naik, \\ Ankita Deshmukhe \\ NMIMS University, India
}

Correspondence: Pravin K Shende, Shobhaben Pratapbhai Patel School of Pharmacy and Technology Management SVKM?s, NMIMS University, Vile Parle (West), Mumbai, India, Tel -42331931, Fax -26185353, Email shendeppravin94@gmail.com

Received: November II, 2016 | Published: December 22, 2016
Abbreviations: PEG, polyethylene glycol; NSAIDs, nonsteroidal anti inflammatory drugs; SD, standard deviation; HD, hemodialysis; SL, spinal length; FD, ferredoxin; PM, promethium; FTIR, fourier transform infrared spectroscopy; DSC, differential scanning calorimetry

\section{Introduction}

Solubility enhancement of poorly aqueous soluble drugs of BCS class II and IV is an important aspect of pharmaceutical research. Solubility associated issues have been elucidated by different technological approaches like solvent dispersion, physical mixture, solid dispersion, hypotrophy, freeze drying during the pharmaceutical product development. ${ }^{1}$ Among them, inclusion complexation is a phenomenon in which one chemical compound act as a host and forms a cavity in second compound which gets entrapped and act as guest. Thus to enhance the solubility of poorly soluble drug, indomethacin was used as a model drug. The polymeric solubilizers like cyclodextrin, polyethylene glycol (PEG) 4000, Lauryl macrogols (gelucire 50/13) were successfully utilized as one of the potential complexing agent which forms inclusion complex with insoluble drugs. ${ }^{2}$ Inclusion complexation is an approach which mainly emphasizes to enhance the solubility of poorly soluble drugs such as NSAIDs (e.g. ibuprofen, mefenamic acid), anti-fungal agents (e.g. ketoconazole), anticancer agents (e.g. cyclophosphamide), anti-epileptic agents (e.g. Phenytoin, carbamazepine), proteins and peptides (e.g. insulin).

NSAIDs, Indomethacin, acts by inhibiting COX I and II enzymes, and its structure shown in Figure 1. It is used in treatment of various conditions like rheumatoid arthritis, pain and headache. In present work an attempt was made to compare the techniques used for solubility enhancement using inclusion complexation for indomethacin. ${ }^{3}$ The objective of present work was to compare the effect of different solubility enhancement by solvent dispersion, physical mixture, hydrotropy, solid dispersion and freeze drying on solubility, in-vitro release and stability studies of indomethacin.

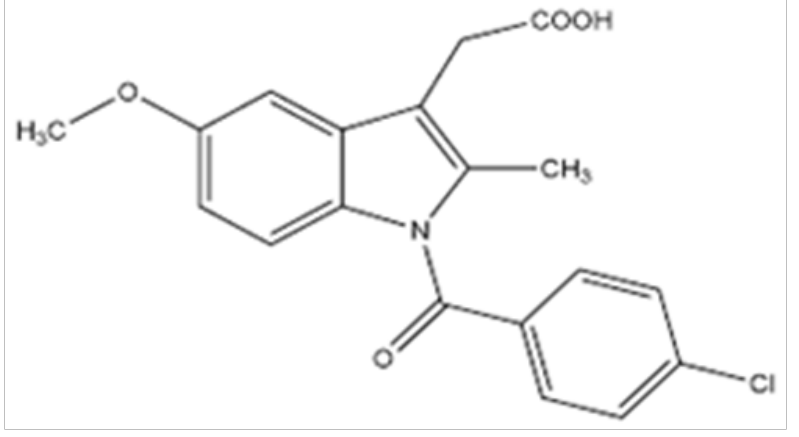

Figure I Structure of Indomethacin. ${ }^{\text {I }}$

\section{Materials and methods}

\section{Materials}

$\beta$-cyclodextrin was gifted by Roquette, France. Indomethacin was purchased from Research Laboratories Fine Chemical Industries, Mumbai, India. All other chemicals and reagents used were of analytical grades.

\section{Methods}

Solvent dispersion: An equivalent quantity of $\beta$-cyclodextrin and ammonia, were heated in a water bath at $100^{\circ} \mathrm{C}$. Eventually the indomethacin was added to the complex and kept under continuous stirring for $24 \mathrm{~h} .{ }^{2}$ The formed dispersion was dried at $50^{\circ} \mathrm{C}$ and stored in glass vial for further use. The formulation was abbreviated as SD.

Physical mixing: The physical mixture was prepared by triturating an equivalent quantity of indomethacin and $\beta$-cyclodextrin in a mortar pestle in molar ratio $1: 1$ for $10 \mathrm{~min}$. The formulation was abbreviated as PM.

Hydrotropy: An equivalent amount of indomethacin was added to $10 \mathrm{~mL}$ of hydrotropic salt solution in $25 \mathrm{~mL}$ of volumetric flask 
containing of polyethylene glycol and water were taken in 4:6 molar ratio. The flask was shaken in a thermostatically controlled water bath at room temperature for $1 \mathrm{~h}$ and the complex was allowed to dry for $24 \mathrm{~h} .{ }^{4}$ The formulation was abbreviated as HD.

Solid dispersion: Indomethacin and PEG 4000 were taken in 1:4 molar ratios in petri dish. The blended mixture was heated above $10^{\circ} \mathrm{C}$ to the melting point of PEG 4000 for 5 min followed by continuous stirring. The dispersion was placed at room temperature for $24 \mathrm{~h}$. The formulation was abbreviated as SL. ${ }^{5}$

Freeze drying: Indomethacin and $\beta$-cyclodextrin were dissolved in methanol in molar ratio of $1: 1$ and freeze dried for $24 \mathrm{~h} .{ }^{6}$ The formulation was abbreviated as FD. The composition of different inclusion complex formulations is shown in Table 1.

Table I Composition of complex formulation

\begin{tabular}{|c|c|c|c|c|c|c|c|}
\hline Methods & Abbreviation & $\begin{array}{l}\text { Indomethacin } \\
\text { (g) }\end{array}$ & $\begin{array}{l}\beta \text {-cyclodextrin } \\
\text { (g) }\end{array}$ & $\begin{array}{l}\text { Ammonia } \\
(\mathrm{mL})\end{array}$ & $\begin{array}{l}\text { PEG } 4000 \\
(\mathrm{~mL})\end{array}$ & $\begin{array}{l}\text { Methanol } \\
\text { (mL) }\end{array}$ & $\begin{array}{l}\text { Water } \\
(\mathrm{mL})\end{array}$ \\
\hline Solvent Dispersion & SD & 4 & 2 & 5 & - & - & - \\
\hline Physical Mixture & PM & I & I & - & - & - & - \\
\hline Hydrotropy & HD & 2 & - & - & 4 & & 6 \\
\hline Solid Dispersion & SL & I & - & - & 4 & - & - \\
\hline Freeze Drying & FD & I & I & - & - & 5 & - \\
\hline
\end{tabular}

Table 2 Particle size and zeta potential of inclusion complex formulations

\begin{tabular}{lll}
\hline Formulation Code & Particle Size $(\mathbf{n m})$ & Zeta Potential $(\mathbf{m V})$ \\
\hline SD & $1417 \pm 2.37$ & $-24.9 \pm 1.18$ \\
PM & $704.7 \pm 1.05$ & $-16.5 \pm 1.23$ \\
HD & $1575 \pm 3.07$ & $-22.2 \pm 1.79$ \\
SL & $479.4 \pm 1.12$ & $-15.3 \pm 1.08$ \\
FD & $638.8 \pm 2.29$ & $-15.7 \pm 1.01$ \\
\hline
\end{tabular}

\section{Characterization}

Particle size: Particle size of indomethacin and formulations (SD, PM, HD, SL and FD) was analyzed using Malvern Zeta Sizer (UK). The samples were analyzed using distilled water. ${ }^{7}$

SEM: The drug and formulations (SD, PM, HD, SL and FD) were coated with a thin gold layer by Sputter coater Machine (SPI Sputter, China) and analyzed by SEM (Hitachi, Japan). ${ }^{8}$

Zeta potential: Zeta potentials of drug and formulations (SD, PM, HD, SL and FD) were analyzed using Malvern Zeta Sizer (UK).

\section{Solubility}

Accurately weighed $5 \mathrm{mg}$ of indomethacin and formulations (SD, PM, HD, SL and FD) were taken in $250 \mathrm{~mL}$ volumetric flask containing distilled water and kept it on rotary shaker (Expo Hi-Tech, Mumbai, India) for $12 \mathrm{~h}$ and analyzed using UV-Visible spectrophotometer (Perkin Elmer, USA) at 267 nm. $^{9}$

\section{Encapsulation efficiency}

Accurately weighed $10 \mathrm{mg}$ of formulation (SD, PM, HD, SL and $\mathrm{FD}$ ) was added in $10 \mathrm{~mL}$ distilled water and allowed to keep for sonication for $30 \mathrm{~min}$. It was then centrifuged for $10 \mathrm{~min}$ and aliquot analyzed using UV-Visible spectrophotometer (Perkin Elmer, USA) at $267 \mathrm{~nm} .{ }^{10}$

\section{In vitro drug release study}

Drug and formulations (SD, PM, HD, SL and FD) were added in a dialysis membrane (Himedia, Mumbai, India.) and placed in $30 \mathrm{ml}$ phosphate buffer $\mathrm{pH} 6.8$ under continuous stirring at 50rpm and temperature $37^{\circ} \mathrm{C}$. The aliquots were withdrawn at scheduled intervals $1,2,16$ and $24 \mathrm{~h}$. The aliquots were subsequently replaced by fresh buffer to maintain sink condition and analyzed using UV-Visible spectrophotometer (Perkin Elmer, USA) at 267nm. ${ }^{11}$

\section{Stability}

The stability study of formulations (SD, PM, HD, SL and FD) was performed in stability chamber (Thermo lab, India) at $40^{\circ} \mathrm{C} / 75 \%$ RH for 6 months as per ICH guideline. On the basis of encapsulation efficiency, solubility, in-vitro release study and stability formulation of solid dispersion was carried out for further studies of FTIR and DSC.

\section{FTIR}

The sample of pure $\beta$-cyclodextrin, indomethacin and formulation of SL were prepared in the form of $\mathrm{KBr}$ pellet method. It was subjected for 32 scans and scanning ranges from 4000-400 cm-1 using FTIR (Perkin Elmer, USA). ${ }^{12}$

\section{DSC}

Indomethacin and formulation SL was sealed in aluminum pan and were heated at a constant rate of $10^{\circ} \mathrm{C} / \mathrm{min}$, under temperature range of $25-200^{\circ} \mathrm{C}$. Thermograms were obtained using DSC (Mettler Toledo, USA). ${ }^{13}$

\section{Results and Discussion}

\section{Particle size and zeta potential}

The particle size was observed in wide-range between $479.4 \pm 1.12$ to $1417 \pm 2.37 \mathrm{~nm}$ and the zeta potential was found to be in between $-15.3 \pm 1.08 \mathrm{mV}$ to $-24.9 \pm 1.18 \mathrm{mV}$ (Table 2 ). The encapsulated drug showed highest efficiency in solid dispersion when complexed with $\beta$-cyclodextrin and the lowest drug was encapsulated in hydrotropy. The size of particles were in order of $\mathrm{HD}>\mathrm{SD}>\mathrm{PM}>\mathrm{FD}>\mathrm{SL}$.

\section{SEM}

Figure 2 crystals seemed to be irregular in shape and size. The solid dispersion of drug and $\beta$-cyclodextrin showed the presence of drug in crystalline form. The crystals were observed to be regular in 
shape, thus forming a crystal lattice oriented in all directions. The appearance of solid dispersion was homogenous with reduction in particle size. The surface morphology of complex also showed a compacted form of drug.

\section{Encapsulation efficiency}

The encapsulation efficiency was done to find out amount of drug entrapped in the cavity using carrier solvent. The encapsulated drug showed highest efficiency in solid dispersion as the hydrophobic drug molecules of indomethacin entered the hydrophilic PEG 4000 structure due to electrostatic attraction. PEG 4000 binds on the cavity of indomethacin that leads to formation of bonds thus exhibiting an effect. The lowest encapsulation efficiency was observed in hydrotropy as there was weak interaction between drug and $\beta$-cyclodextrin that lead to poor bonding, hence the order of encapsulation observed was $\mathrm{SL}>\mathrm{SD}>\mathrm{FD}>\mathrm{PM}>\mathrm{HD}$ as shown in Figure 3.

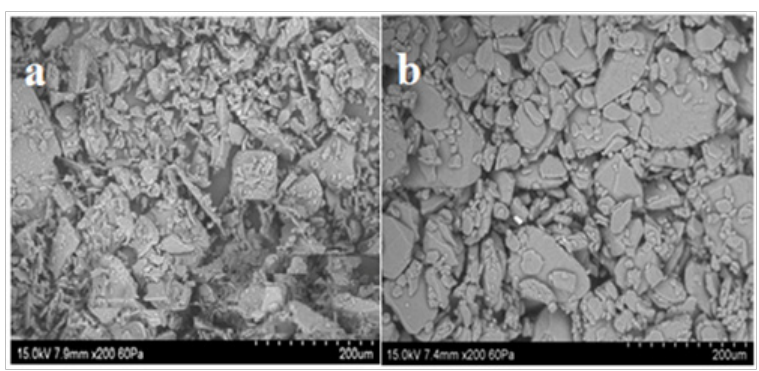

Figure 2 SEM results of Indomethacin (a) and Solid Dispersion (b).

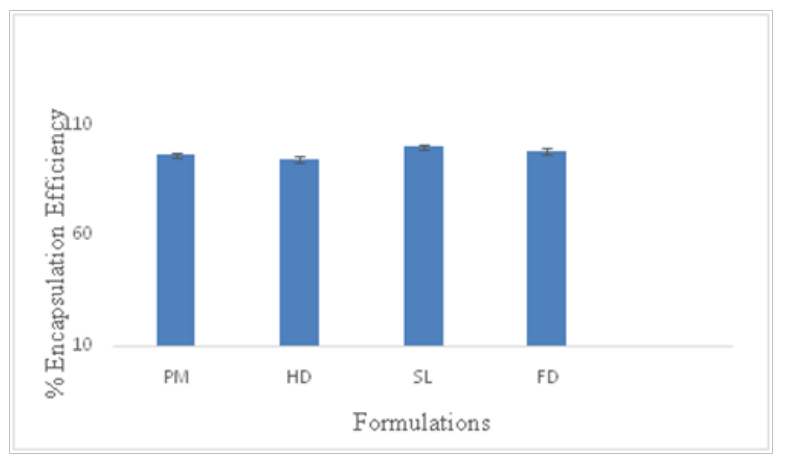

Figure 3 Encapsulation efficiency of indomethacin in different inclusion complex formulation.

\section{Solubility studies}

The results of solubility studies of freeze drying, solvent dispersion, physical mixture prepared by various methods revealed that there was sufficient increase in solubility, but drastic solubility improvement was seen with PEG 4000 in solid dispersion that lead to slow drug release from the cavity. Due to sufficient reduction in particle size, the entrapment was maximum that led to increase in solubility, however hydrotropy showed the least solubility result as the hydrophilic carrier formed weak bonds with the drug thus formed weak complex. The order of solubility was found to be $\mathrm{SL}>\mathrm{SD}>\mathrm{FD}>\mathrm{PM}>\mathrm{HD}$ (Figure 4). The solubility of formulations was found to be increased as compared to pure drug due to decreased in particle size, improved wettability and presence of polymers and excipients.

\section{In vitro drug release study}

It was observed that the drug releases slowly from $\beta$-cyclodextrin cavity over a definite period of time. The rate of complex formation and hydration leads to greater availability of drug. Cyclodextrins can significantly increase the dissolution rate of poorly soluble substances, which on the other hand, can be reduced if the substance is in ionized form. The drug release also showed significant increase when complexed with PEG 4000 in ratio $1: 4,{ }^{3}$ as solvent left the surface rapidly to get dispersed in the dissolution media in bulk and lead to increase in solubility of indomethacin. Unionized drugs do usually form more stable complexes than their ionic counterparts. However, ionization of a drug increases its apparent intrinsic solubility resulting in enhanced complexation. Release studies were carried out using phosphate buffer $\mathrm{pH} 6.8$ to compare the percent release of complexes using different methods. The drug release was in order of $\mathrm{SL}>\mathrm{PM}>\mathrm{HD}>\mathrm{SD}>\mathrm{FD}$ (Figure 5). On the basis of in vitro drug release study it can be concluded that solid dispersion shows the better drug release. It can be concluded that polymer responsible for eroding the drug showed optimum results when taken in 1:4 as ratio in solid dispersion.

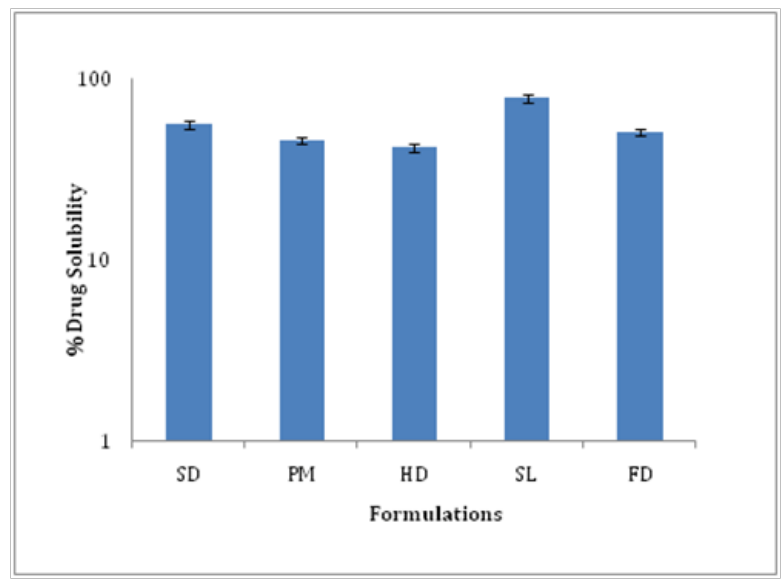

Figure 4 Drug solubility study of indomethacin in different formulations.

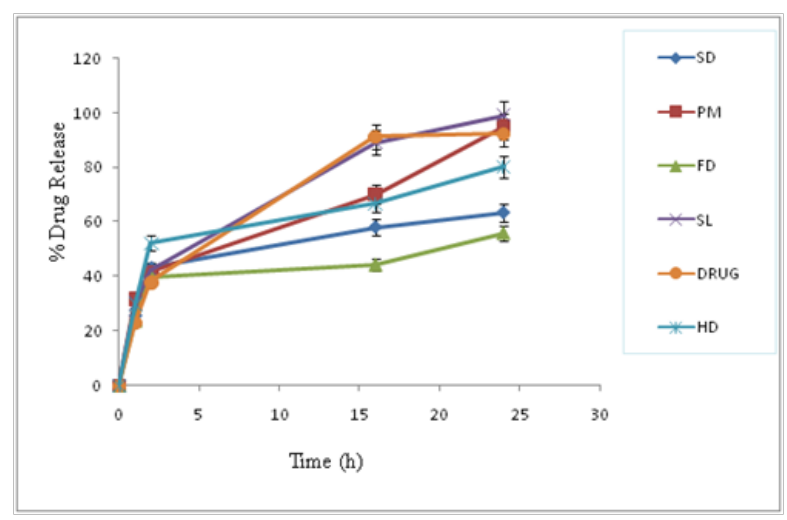

Figure $\mathbf{5}$ In vitro drug release of different formulations.

On the basis of encapsulation efficiency, solubility, in-vitro release study and stability formulation of solid dispersion was carried out for further studies of FTIR and DSC.

\section{FTIR}

FTIR spectra of indomethacin and SL are shown in the Figure 6. The characteristic peaks were compared with the IR spectrum of selected samples. The result of FTIR revealed that there was interaction between drug and polymers. The spectrum of indomethacin exhibit a characteristic peak at $3735 \mathrm{~cm}^{-1}$ (OH stretch), $1899 \mathrm{~cm}^{-1}$ (aryl ketone stretch) and $1507 \mathrm{~cm}^{-1}(-\mathrm{NO}$ stretch) while the shift in spectra of solid dispersion was observed due to interaction of drug and $\mathrm{PEG}$ that exhibit peaks at $2662 \mathrm{~cm}^{-1}(\mathrm{CH}$ stretch $), 1685 \mathrm{~cm}^{-1}(\mathrm{C}=\mathrm{O}$ stretch $)$ and $1522 \mathrm{~cm}^{-1}(\mathrm{NO}$ stretch) respectively. 


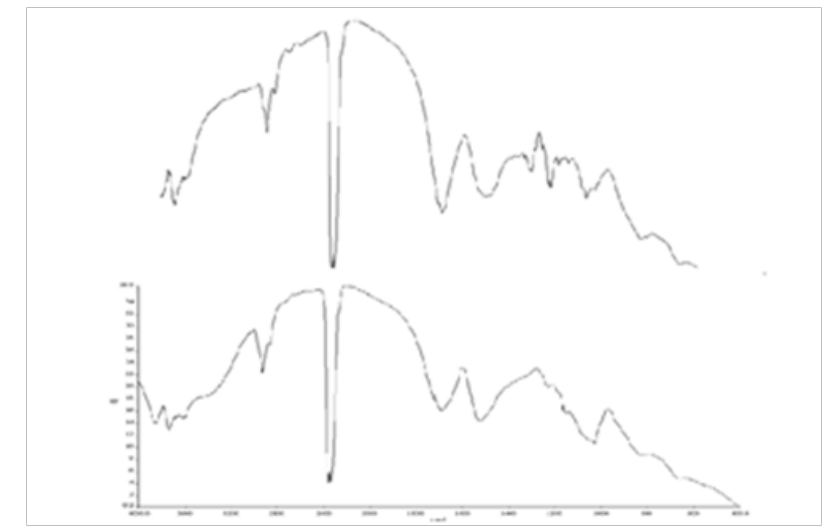

Figure 6 FTIR spectra of indomethacin (a) and solid dispersion (b).

\section{DSC}

The formulation SL and indomethacin thermograms showed an event at $75^{\circ} \mathrm{C}$ and $150^{\circ} \mathrm{C}$ respectively (Figure 7). The drug peak disappears in the complex indicates a strong complexation due to entrapment of drug in the cavity. The change in melting point suggested that the complex has been formed and was a mixture of drug and polymer.

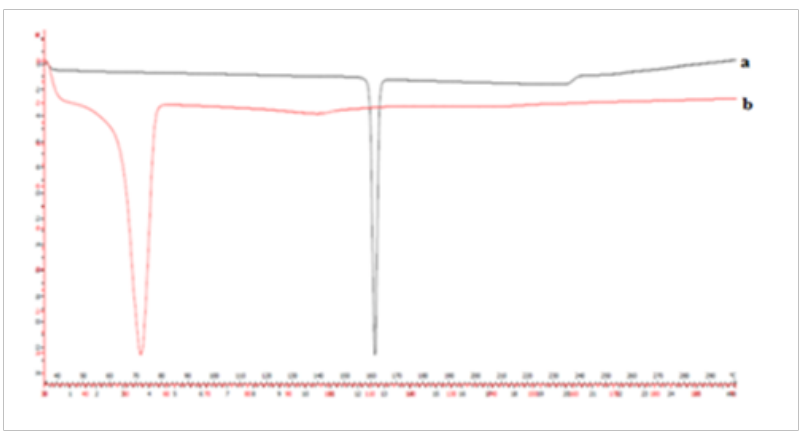

Figure 7 DSC of indomethacin (a) and solid dispersion (b).

\section{Conclusion}

The effect of different inclusion complexation methods solvent dispersion, physical mixture, hydrotropy, solid dispersion and freeze drying on solubility, in-vitro release and stability studies of indomethacin showed satisfactory results in solid dispersion compared to other methods. The complexation is a promising alternative for formulation of water insoluble drugs when compared to the standard dosage forms of indomethacin. Solid dispersion method emphasizes its importance in enhancing the solubility bioavailability, controlled release and stability of indomethacin.

\section{Acknowledgments}

None.

\section{Conflicts of interest}

Author declares there are no conflicts of interest.

\section{Funding}

None.

\section{References}

1. Singh R, Bharti N, Mada J, et al. Characterization of Cyclodextrin Inclusion Complexes - A Review.Journal of Pharmaceutical Science and Technology. 2010;2(3):171-183.

2. Badry M, El Feith G, Fathy M. Improvement of solubility and dissolution rate of indomethacin by solid dispersions in Gelucire 50/13 and PEG4000. Saudi Pharm J. 2009;17(2):217-225.

3. Lim HP, Holluand H, Fahmy R, et al. Solubility enhancement of poorly water soluble drug using a novel polymeric solubilizer(Solupus), University of Maryland, Baltimore, USA. 2009.

4. Caselia R, Wiliams DA, Jambhekar SS. Solid state $\beta$-cyclodextrin complexes containing indomethacin, ammonia and water. International Journal of Pharmaceutics. 1998;165(1):15-22.

5. Etman M, Nada AH. Hydrotropic and cosolvent solublization of Indomethacin. Acta Pharmaceutica. 1999;49(4):291-298.

6. Sharma M, Sharma S. Preparation Physicochemical Characterization, Dissolution, Formulation and Spectroscopic studies of $\beta$-Cyclodextrins Inclusion Complex. International Journal of Chem Tech Research. 2011;3(1):101-104.

7. Janamala RS, Valluru R, Thudi A, et al. Solubility Enhancement of Indomethacin by Solid Dispersion Technique and Development of Fast dissolving tablet. International Research Journal of Pharmacy. 2012;3(12):145-150.

8. Shende PK, Gaud RS, Bakal R, et al. Effect of inclusion complexation of meloxicam with $\beta$-cyclodextrin-and $\beta$-cyclodextrin based nanosponges on solubility, in vitro release and stability studies. Colloids surf $B$ Biointerfaces. 2015;136:105-110.

9. Kapoor B, Kaur R, Kour S, et al. Solid Dispersion: An Evolutionary Approach for Solubility Enhancement of Poorly Water Soluble Drugs. Indo American Journal of Pharmaceutical Research. 2014;4(6):1-16.

10. Saeedia M, Akbari J, Semnani KM, et al. Enhancement of Dissolution Rate of Indomethacin Using Liquisolid Compacts.Iran J Pharm Res. 2011;10(1):25-34.

11. Gina S, EL-Fekya, Sally T, et al. Preparation, in-vitro and invivo evaluation of oral indomethacin and HP- $\beta$-cyclodextrin chitosan loaded nanoparticles. International Journal of Pharmacy and Pharmaceutical Sciences. 2013;5(4):1-8.

12. Yadav VB, Yadav AV. Improvement of Solubility and Dissolution of Indomethacin by Liquisolid and Compaction Granulation Technique. $J$ Pharma Sci \& Research. 2009;1(2):44-51.

13. Kataria MK, Bhandari A. Solubility and Dissolution Enhancement: Technologies and Research Emerged. Journal of Biological \& Scientific Opinion. 2013;1(2):105-116.

14. Shende P, Gaud RS, Deshmukh k, et al. Influence of Different Techniques on Formulation and Comparative Characterization of Inclusion Complexes of ASA with $\beta$-cyclodextrin and Inclusion Complexes of ASA with PMDA cross-linked $\beta$-cyclodextrin Nanosponges. Journal of Inclusion Phenomena and Macrocyclic Chemistry. 2012;74(1):447-454. 